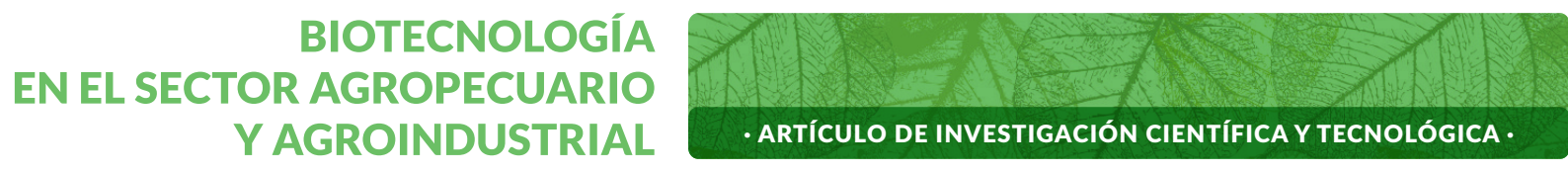

Vol. 18 No 2 · Julio - Diciembre 2020 • ISSN - 1692-3561 · ISSN-e 1909-9959 · DOI: http://dx.doi.org/10.18684

\title{
Caracterización del epicarpio de guayaba (Psidium guajava L.) como alternativa natural para uso en productos alimenticios procesados*
}

\author{
Characterization of guyaba epicarp \\ (Psidium guajava L.) as a natural alternative \\ for use in processed food products
}

\section{Caracterização do epicarpo de guyaba (Psidium guajava L.) como alternativa natural para uso em produtos alimentícios processados}

\author{
VIVIANA ANDREA VELASCO-ARANGO ${ }^{1}$, ALICIA ALEJANDRA BERNAL-MARTÍNEZ², \\ LUIS EDUARDO ORDÓÑEZ-SANTOS ${ }^{3}$, JOSÉ IGOR HLEAP-ZAPATA ${ }^{4}$
}

Historial del Artículo

Recibido para evaluación: 8 de Octubre 2019.

Aprobado para publicación: 4 de Mayo 2020.

* Proyecto de investigación de origen: "Valorización agroindustrial de pigmentos carotenoides extraídos de residuos de papaya (Carica papaya L.) y guayaba (Psidium guajava) como colorante natural en salchichas Frankfurt". Financiación: Proyecto HERMES 43547 Universidad Nacional de Colombia - Sede Palmira. Culminación: 30 de marzo de 2020

1 Universidad Nacional de Colombia Sede Palmira, Facultad de Ingeniería y Administración, Grupo de Investigación en Manejo y Agroindustrialización de Productos de Origen Biológico. Candidata a Magister en Ingeniería Agroindustrial. Palmira, Colombia. ORCID: 0000-00032514-1267

2 Universidad Nacional de Colombia Sede Palmira, Facultad de Ingeniería y Administración, Grupo de Investigación en Manejo y Agroindustrialización de Productos de Origen Biológico. Ingeniera Agroindustrial. Palmira, Colombia. ORCID: 0000-0002-6153-4773

3 Universidad Nacional de Colombia Sede Palmira, Facultad de Ingeniería y Administración, Grupo de Investigación en Procesos Agroindustriales (GIPA). Ph.D. en Ciencias Biológicas. Palmira, Colombia. ORCID: 0000-0002-5958-027X

4 Universidad Nacional de Colombia Sede Palmira, Facultad de Ingeniería y Administración, Grupo de Investigación en Manejo y Agroindustrialización de Productos de Origen Biológico. Ph.D. en Ingeniería de Alimentos. Palmira, Colombia. ORCID: 0000-0001-9692-5443 


\section{RESUMEN}

Dentro de las frutas tropicales, la guayaba (Psidium guajava L.) es una de las más consumidas gracias a sus excelentes propiedades organolépticas y funcionales, rica en compuestos bioactivos. Su aprovechamiento agroindustrial genera gran cantidad de subproductos, incluido el epicarpio, el cual representa aproximadamente entre el 25 y el $30 \%$ del peso de la fruta. El objetivo de la presente investigación fue determinar, en el epicarpio de la guayaba, las propiedades fisicoquímicas, las coordenadas de color y caracterizarlo en su contenido de compuestos carotenoides y fenólicos, así como también determinar la actividad antioxidante del mismo. Se elaboró la harina de epicarpio de guayaba (HEG) y con base en métodos aprobados se le determinó rendimiento en peso, $\mathrm{pH}$, acidez titulable, sólidos solubles, contenido de humedad, actividad de agua y parámetros de color, así como los compuestos bioactivos anotados. Los resultados, para los parámetros fisicoquímicos fueron altos y el contenido de compuestos carotenoides para las diferentes fracciones fluctuó entre 9,414 para la fracción de $\alpha$-caroteno y 10,894 para la fracción de $\beta$-criptoxantina. La actividad antioxidante, arrojó un valor de $62,281 \%$ y el contenido de compuestos fenólicos fue de 24,948 mg EAG/g. Se concluye que la HEG puede ser utilizada como fuente de compuestos bioactivos naturales en la industria alimenticia.

\section{ABSTRACT}

Among tropical fruits, guava (Psidium guajava L.), is one of the most consumed thanks to its excellent organoleptic and functional properties, rich in bioactive compounds. Its agroindustrial use generates a large number of by-products, including the epicarp, which represents approximately 25 to $30 \%$ of the fruit's weight. The objective of the present investigation was to determine, in the epicarp of guava, the physicochemical properties, the color coordinates and characterize it in is content of carotenoid and phenolic compounds, as well as to determine its antioxidant activity. Guava epicarp flour (GEF) was made and based on approved methods, yield in weight, $p H$, titratable acidity, soluble solids, moisture content, water activity and color parameters was determined, as well as the bioactive compounds noted. The results for the physicochemical parameters were high and the content of carotenoid compounds for the different fractions fluctuated between 9,414 for the $\alpha$-carotene fraction and 10,894 for the $\beta$-cryptoxanthin fraction. The antioxidant activity showed a value of $62,281 \%$ and the content of phenolic compounds was $24,948 \mathrm{mg} E A G / g$. It is concluded that GEF can be used as a source of natural bioactive compounds in the food industry.

Correspondencia: jihleapz@unal.edu.co

Cómo citar este artículo: VIVIANAANDREA VELASCO-ARANGO, ALICIAALEJANDRA BERNAL-MARTÍNEZ, LUIS EDUARDO ORDÓÑEZ-SANTOS, JOSÉ IGOR HLEAPZAPATA. Evaluación del efecto antifúngico de metabolitos de cultivos bioprotectores: aplicación en derivados lácteos. Revista Biotecnología en el Sector Agropecuario y Agroindustrial,18(2),2020.26-35, DOI:http://dx.doi.org/10.18684/BSAA(18)26-36

\section{PALABRAS CLAVE:}

Antioxidantes, Colorantes naturales, Compuestos fenólicos, Frutas tropicales.

\section{KEY WORDS:}

Antioxidants, Natural colorants, Phenolic compounds, Tropical fruits.

\section{PALAVRAS-CHAVE:}

Antioxidantes, Corantes naturais, Compostos fenólicos, Frutas tropicais 


\section{RESUMO}

Entre as frutas tropicais, a goiabeira (Psidium guajava L.) é uma das mais consumidas, graças às suas excelentes propriedades organolépticas e funcionais, ricas em compostos bioativos. Seu uso agroindustrial gera um grande número de subprodutos, incluindo o epicarpo, que representa aproximadamente 25 a 30\% do peso da fruta. O objetivo da presente investigação foi determinar, no epicarpo de goiaba, as propriedades físico-químicas, as coordenadas de cor e caracterizá-la em seu conteúdo de compostos carotenóides e fenólicos, bem como determinar sua atividade antioxidante. A farinha de epicarpo de goiaba (FEG) foi confeccionada e baseada em métodos aprovados, foi determinado o rendimento em peso, $\mathrm{pH}$, acidez titulável, sólidos solúveis, umidade, atividade de água e parâmetros de cor, bem como os compostos bioativos observados. Os resultados para os parâmetros físico-químicos foram elevados e o conteúdo de compostos carotenóides para as diferentes frações flutuou entre 9,414 para a fração $\alpha$-caroteno e 10,894 para a fração $\beta$-criptoxantina. A atividade antioxidante apresentou um valor de $62,281 \%$ e o teor de compostos fenólicos foi de 24,948 mg EAG / g. Conclui-se que o FEG pode ser usado como fonte de compostos bioativos naturais na indústria alimentícia.

\section{INTRODUCCIÓN}

En la actualidad, el alto desarrollo de la industria alimenticia ha conllevado a la generación de gran cantidad de residuos, tanto biodegradables como no biodegradables, lo cual ha obligado al perfeccionamiento e implementación de nuevas técnicas o métodos para el aprovechamiento de los mismos [1, 2, 3, 4]. En particular, los residuos resultantes de la agroindustria hortofrutícola, generalmente han sido utilizados para la fabricación de productos de valor agregado destinados básicamente a la alimentación animal o a la elaboración de insumos para la producción agrícola, lo que ha permitido minimizar la carga de estos sobre el medio ambiente y, por ende, aliviar los resultados negativos ambientales tales como propagación de plagas, malos olores, contaminación de suelos y cuerpos de agua, entre otros [5]. Sin embargo, una gran cantidad de estos subproductos terminan desechados, lo que implica, además de lo anotado referente al medio ambiente, pérdidas económicas considerables para los productores y comercializadores.
La guayaba rosada (Psidium guajava L) es conocida como la "manzana de los trópicos" debido a sus atractivas características de color, aroma, sabor y propiedades funcionales. Cultivada por primera vez en América Central, la guayaba rosada se ha convertido en un importante cultivo comercial en muchos países del mundo, incluidos Pakistán, Brasil, México, Egipto, India, Indonesia y Colombia entre otros [6]. Durante el año 2016 se cosecharon en Colombia 11.131 ha de guayaba, con una producción total de 119.161 ton, distribuida en 12 departamentos dentro de los cuales se destacan Santander, Meta, Valle del Cauca, Boyacá, Antioquia y Caldas [7].

Perteneciente al grupo de las frutas denominadas tropicales, de pulpa jugosa color rosado, ha sido considerada una fuente importante de carotenoides, pectina, fibra dietética, y otros fitoquímicos como el ácido ascórbico, el ácido elágico y las antocianinas $[6,8,9,10]$.

Con fines comerciales, la guayaba se procesa en forma de pulpa, jugos, néctares, mermeladas y concentrados, pero estos productos involucran aproximadamente la utilización del 70-75\% de la fruta [9]. La agroindustrialización de la guayaba presupone el descarte de sus subproductos (25-30\% de la fruta), lo cual conduce a los problemas ambientales anteriormente anotados. Estos residuos (epicarpio y semillas) han sido aprovechados para la elaboración de compuestos antimicrobianos, producción de etanol, biomasa ácido-láctica y ácido láctico $[11,12]$. Sin embargo, el epicarpio de algunas frutas presenta gran importancia a nivel agroindustrial ya que contiene pigmentos carotenoides que pueden ser utilizados como fuente importante de colorantes naturales o de provitamina A $[13,14]$.

Los carotenoides son pigmentos naturales ampliamente distribuidos en múltiples productos de origen vegetal y animal y son los responsables de la coloración amarilla, naranja o roja de diversas frutas, raíces, flores, invertebrados, peces y pájaros entre otros $[15,16,17]$. Diversos estudios han demostrado que la ingesta de carotenoides se relaciona con una disminución del riesgo de padecer diferentes tipos de cáncer, enfermedades cardiovasculares, cataratas, degeneración macular, enfermedades neurológicas, inflamatorias y desórdenes inmunológicos [6,9].

En múltiples investigaciones se han estudiado, separado e identificado como carotenoides mayoritarios de la guayaba el fitoflueno, el $\beta$-caroteno y en mayor propor- 
ción el licopeno, haciendo de la guayaba una excelente fuente de estos componentes [6, 9, 10, 18, 19, 20, 21].

En este orden de ideas, los pigmentos carotenoides se constituyen como una fuente importante de color para ser aplicados a diferentes matrices alimenticias en sustitución de colorantes artificiales o sintéticos. En particular, la industria cárnica en la formulación de múltiples productos adiciona nitratos y nitritos, que además de cumplir la función de actuar como conservantes y antioxidantes, juegan un papel preponderante en el desarrollo de la coloración de los mismos. Sin embargo, en las últimas décadas se ha discutido el uso de ellos, ya que su consumo en cantidades altas conlleva a problemas de intoxicación y desarrollo de enfermedades catastróficas [22, 23, 24, 25]. El objetivo del presente estudio fue caracterizar la harina de epicarpio de guayaba y determinar sus componentes carotenoides, fenólicos y su actividad antioxidante, con fines de ser aprovechada como aditivo en productos alimenticios procesados.

\section{MÉTODO}

El estudio se llevó a cabo en el Laboratorio de Tecnología de Frutas y Hortalizas de la Universidad Nacional de Colombia - Sede Palmira, ubicado en el municipio de Palmira, Valle del Cauca, Colombia, ubicado a $1001 \mathrm{msnm}$ y con una temperatura promedio de $24^{\circ} \mathrm{C}$. Su cabecera está situada a $3^{\circ} 31^{\prime} 48^{\prime \prime}$ de latitud norte y $76^{\circ} 81^{\prime} 13^{\prime \prime}$ de longitud oeste. Las guayabas se adquirieron en un mercado local especializado en venta de frutas en grado de madurez para consumo en fresco. Con la ayuda de un cuchillo en acero inoxidable, se retiró el epicarpio, procurando eliminar los residuos de pulpa adheridos. Éste se pesó y se empacó en bolsas de polietileno selladas al vacío, para lo cual se utilizó una máquina selladora manual EGARVAC S.C.P. Basic B (Vacarisses, Barcelona, España). Las bolsas selladas se sometieron a conservación bajo congelación $\left(-30 \pm 1^{\circ} \mathrm{C}\right)$ durante 24 horas. Posteriormente fueron sometidas a secado por liofilización utilizando un aparato liofilizador LABCON$\mathrm{CO}$, de $18 \mathrm{~L}$ de capacidad, a presión de vacío a 0,120 mbar y temperatura del condensador $-80^{\circ} \mathrm{C}$ durante 24 horas. Finalmente, el epicarpio seco se molió en un molino IKA M-20s3, USA hasta obtener una harina con diámetro de gránulo promedio de $0,25 \mathrm{~mm}$, la cual se empacó en bolsas al vacío y se mantuvo a temperatura de refrigeración $\left(5 \pm 1^{\circ} \mathrm{C}\right)$ hasta el momento de realizar los respectivos análisis.

\section{Parámetros fisicoquímicos}

La harina de epicarpio de guayaba (HEG) se sometió a los siguientes análisis: rendimiento en peso (\%), $\mathrm{pH}$, sólidos solubles ( ${ }^{\circ}$ Brix), acidez titulable (\%), parámetros de color ( $\left.L^{*}, a^{*}, b^{*}, C, h\right)$, contenido de humedad (\%) y actividad de agua $\left(\mathrm{A}_{\mathrm{w}}\right)$. De la misma forma, se determinó el contenido de carotenoides, la actividad antioxidante y el contenido de compuestos fenólicos. Todas las pruebas se realizaron por triplicado.

Rendimiento en peso (\%). Se midió el peso del epicarpio tanto en fresco como después del proceso de liofilización y se calculó el rendimiento mediante la siguiente ecuación:

$R=\frac{\omega 1}{\omega 2} \times 100$

Donde:

R: Rendimiento (\%)

$\omega 1:$ Peso del epicarpio después de la liofilización (g)

w2: Peso del epicarpio antes de la liofilización (g)

Determinación del pH. Con la ayuda de un pH-metro (MP 230, Metter Toledo, Suiza) calibrado con soluciones tampón de $\mathrm{pH} 7$ y pH 4 se midió el pH de la salchicha, para lo cual a $1 \mathrm{~g}$ de la muestra se le adicionaron $10 \mathrm{~mL}$ de agua destilada hasta obtener una solución homogénea (Norma Técnica Colombiana NTC - 4592) [26].

Determinación de sólidos solubles ( ${ }^{\circ}$ Brix). A $10 \mathrm{~g}$ de la muestra se le adicionó agua destilada en relación 1:10. Se mezcló con agitación permanente en un beaker de $250 \mathrm{~mL}$ hasta obtener una mezcla homogénea. Sobre el prisma inferior del refractómetro (POCKET ATAGO PAL - 1, Japón) se colocó una alícuota de la mezcla y se hizo la respectiva medición. El resultado se expresó en ${ }^{\circ}$ Brix de acuerdo con la Norma Técnica Colombiana NTC - 4624 [26].

Determinación de la acidez titulable (\% Acidez). En un beaker se colocó $1 \mathrm{~g}$ de la muestra y se mezcló con $10 \mathrm{~mL}$ de agua destilada, hasta obtener una mezcla homogénea. Se agregaron tres gotas de fenolftaleína como indicador, y se tituló con una solución $0,1 \mathrm{~N}$ de $\mathrm{NaOH}$ hasta obtener un $\mathrm{pH} 8,1-8,2$ según la Norma 
Técnica Colombiana NTC - 4623 [26]. El porcentaje de acidez se calculó mediante la siguiente ecuación:

$\%$ Acidez $=\frac{(m l N a O H \times 0,1 \times F . E q v t)}{m l \text { muestra }} \times 100 \quad$ (Ec. 2)

Determinación de los parámetros de color. Las mediciones se realizaron en un colorímetro MICOLTA CHROMA METER CR-400, Japón), a través del cual se evaluaron los parámetros CIELab de la Comisión Internacional de lluminación. Se utilizó un iluminante D65 y un observador a $2^{\circ}$ (equipo calibrado con una placa de cerámica blanca con valores de referencia $Y$ $=89,5, x=0,3176$ y $y=0,3340)$. Con base en estos parámetros se calcularon la tonalidad (h) y la cromaticidad (C) utilizando las siguientes ecuaciones:

$C=\left(a *^{2}+b *^{2}\right)^{1 / 2}$

$h^{\circ}=\arctan \left(b^{*} / a^{*}\right)$

Determinación del contenido de humedad (\%). Se pesaron $0,5 \mathrm{~g}$ de la muestra y se colocaron en un crisol a $105^{\circ} \mathrm{C}$ en un analizador halógeno de humedad HB43-S Metter Toledo, Suiza de acuerdo con el método AOAC 925.09 [27]. El contenido de humedad se expresó en porcentaje de peso húmedo.

Determinación de la actividad de agua $\left(\mathrm{A}_{\mathrm{w}}\right)$. Con la ayuda de un equipo para medir actividad de agua (AquaLab, 4te, USA) y de acuerdo con la norma AOAC 978.19 [28] se determinó la actividad de agua de las muestras analizadas.

Determinación del contenido de carotenoides. EI contenido de carotenoides totales en la HEG se realizó de acuerdo a lo propuesto por Ordoñez et al. [15]. $0,1 \mathrm{~g}$ de la muestra se colocaron en un tubo de ensayo recubierto con papel aluminio y se le adicionaron $7 \mathrm{~mL}$ de una mezcla de etanol/hexano (4:3 v/v). El tubo se colocó a $16^{\circ} \mathrm{C}$ durante una hora agitándolo permanentemente, posteriormente se le adicionó 1 $\mathrm{mL}$ de agua destilada y se agitó por 5 minutos más. Seguidamente se centrifugó a 4500 rpm durante 10 minutos, se tomaron $3 \mathrm{~mL}$ de la fase orgánica y se realizaron lecturas de absorbancia en un espectrofotómetro (Jenway, 6320D, Inglaterra) utilizando hexano como blanco, a longitudes de onda 450, 441, 451 y $472 \mathrm{~nm}$. La concentración (mg/g de muestra) se calculó utilizando los siguientes coeficientes de extinción E\% en hexano 2560, 2800, 2460, 2480 y 3450 respectivamente para $\beta$-caroteno, $\alpha$-caroteno, $\beta$-criptoxantina, zeaxantina y licopeno, mediante la siguiente ecuación (ecuación de Lambe-Beer):

$$
\text { C.T. }=\frac{A * V * 10^{3}}{E^{1 \% * W}}
$$

Donde:

A: Absorbancia a determinada longitud de onda (450 $\mathrm{nm}$ para $\beta$-caroteno, $444 \mathrm{~nm}$ para $\alpha$-caroteno, 451 $\mathrm{nm}$ para $\beta$-criptoxantina, $451 \mathrm{~nm}$ para zeaxantina y $472 \mathrm{~nm}$ para licopeno.

$\mathrm{V}$ : volumen del extracto en $\mathrm{mL}$.

W: peso de la muestra en gramos.

E 1\%: coeficiente de extinción para una solución al $1 \%$ en hexano.

Determinación de la actividad antioxidante. El proceso se desarrolló de acuerdo con la metodología propuesta por Martínez et al. [29]. La actividad antioxidante se expresó como el porcentaje de inhibición del radical DPPH (2,2,-diphenyl-1-picrylhydrazyl radicals, marca Sigma-Aldrich Chemical). Se pesaron 0,25 g de la muestra de HEG y se colocaron en un Erlenmeyer cubierto con papel aluminio. Se le adicionaron $25 \mathrm{~mL}$ de metanol y se agitó durante un minuto, posterior a lo cual la solución se filtró hasta obtener el extracto. Se tomó $1 \mathrm{~mL}$ del extracto y $2 \mathrm{~mL}$ de DPPH 0,1 mM recién preparado. La muestra y el control (sin adición de DPPH) se dejaron en reposo durante una hora en un sitio oscuro y a temperatura ambiente. Finalmente, con la ayuda de un espectrofotómetro Jenway 6320D, Inglaterra, se leyeron las absorbancias, para la muestra y para el control, a una longitud de onda de $517 \mathrm{~nm}$. El porcentaje de actividad antioxidante (AA\%), se calculó usando la siguiente ecuación:

$\% A A=\frac{A_{517 \text { Control }}-A_{517 \text { Muestra }}}{A_{517 \text { Control }}} \times 100$

Determinación de los compuestos fenólicos. El contenido de fenoles se determinó de acuerdo con el método de Folin-Ciocalteu descrito por Singleton et al. [30]. $0,5 \mathrm{~g}$ de HEG se colocaron en un Erlenmeyer de 50 $\mathrm{mL}$ cubierto con papel aluminio y se le adicionaron 10 $\mathrm{mL}$ de una mezcla etanol-agua en relación 80:20 (v/v). 
La mezcla se mantuvo a temperatura ambiente en constante agitación por tres minutos. Seguidamente, la mezcla se sometió al proceso de ultrasonido, en un equipo Ultrasonic Cleaner-DC PowerPowerFull-Hagavish, Israel durante 20 minutos a $25^{\circ} \mathrm{C}$. Posteriormente, la mezcla se filtró y $0,5 \mathrm{~mL}$ del filtrado se mezclaron con agua destilada y $0,5 \mathrm{~mL}$ del reactivo de Folin-Ciocalteu. Después de tres minutos de reacción a temperatura ambiente se le adicionó $1 \mathrm{~mL}$ de carbonato de sodio anhídrido al $20 \%$ y se colocó en un baño térmico programado a $45^{\circ} \mathrm{C}$ por 15 minutos. Después de enfriada la muestra se hicieron lecturas de absorbancia en un equipo Spectrophotometer Jenway 632D, USA, a una longitud de onda de $765 \mathrm{~nm}$. Se realizó una curva de calibración con ácido gálico (patrón) y los resultados fueron expresados en $\mathrm{mg}$ de ácido gálico equivalentes por gramo de extracto de HEG.

\section{Análisis estadístico}

El utilizó un diseño aleatorio simple de un factor y un tratamiento. Para comparar las diferencias entre medias de los diferentes parámetros analizados se utilizó una prueba Tukey, con un nivel de significancia del $5 \%$. Todos los análisis se realizaron por triplicado y se registran como media \pm desviación estándar.

\section{RESULTADOS}

Los resultados de los parámetros fisicoquímicos referentes a la HEG se muestran en el cuadro 1. El rendimiento en peso de la muestra analizada fue del $11,5 \%$. Con relación al pH, la HEG presentó un valor de 4,23 siendo ligeramente ácido lo que garantiza la no proliferación de microorganismos patógenos y, por lo tanto, la inocuidad del producto obtenido. Este valor de $\mathrm{pH}$ en la HEG concuerda con lo reportado por Ordóñez-Santos et al. (pH 4,29) [15], quienes trabajaron con residuos de guayaba, igualmente con lo presentado por Vasconcelos et al., $(\mathrm{pH} 3,99)$ quienes trabajaron con pulpa de arazá (Psidium guineense) [31], pero superior a lo presentado por Rojas-Garbanzo et al. ( $\mathrm{pH} 2,72-2,77)$ en un estudio de caracterización de los compuestos fitoquímicos de la guayaba [20] y lo encontrado por Formiga et al. $(\mathrm{pH} 3,69)$ quienes analizaron las características de la piel de guayaba de la variedad "Pedro Sato" [32]. Las diferencias del valor de $\mathrm{pH}$ anotadas probablemente se deben a la variedad y al grado de madurez de la guayaba utilizada en las diferentes investigaciones.
El resultado obtenido para el contenido de sólidos solubles ( ${ }^{\circ} \mathrm{Brix}$ ) en la HEG elaborada mostró un valor inferior a lo presentado por Martínez-Ortiz et al. $\left(10,5^{\circ} \mathrm{Brix}\right)$ [33], quienes trabajaron con guayabas recubiertas con una película a base de harina de chayote y ácido ascórbico, así como también por lo mostrado por Zambrano-Zaragoza et al. (9,01 ${ }^{\circ}$ Brix) [34] adicionando nanopartículas lipídicas como recubrimiento en guayabas y por Alamar et al. (7,0 $\left.{ }^{\circ} \mathrm{Brix}\right)$ [35] quienes trabajaron con guayabas congeladas. Los resultados para los sólidos solubles encontrados por los autores anotados muestran valores superiores debido, probablemente, a que éstos trabajaron con la pulpa de la fruta, mientras que esta investigación se llevó a cabo con el epicarpio de la fruta en donde evidentemente el contenido de sólidos solubles es menor. Con relación a la acidez titulable, el valor obtenido fue similar a lo reportado por Moon et al. $(0,50 \%)$ [36] quienes trabajaron con 27 variedades de guayaba en diferentes estados de maduración, y por Murmu y Mishra (0,49\%) [37] quienes adelantaron la respectiva investigación con guayaba empacada bajo diferentes condiciones de almacenamiento, pero ligeramente inferior a lo obtenido por Silva et al. $(0,75 \%)$ [38] quienes investigaron con guayaba recubierta con quitosano.

Dentro de los parámetros de calidad referidos a una matriz alimenticia, el color juega un papel prepon-

Cuadro 1. Propiedades fisicoquímicas para la harina de epicarpio de guayaba (HEG).

\begin{tabular}{|c|c|}
\hline Parámetros fisicoquímicos & $\begin{array}{c}\text { Harina de epicarpio } \\
\text { de guayaba (HEG) }\end{array}$ \\
\hline Rendimiento en peso (\%) & 11,5 \\
\hline $\mathrm{pH}$ & $4,23 \pm 0,034$ \\
\hline Sólidos solubles ( ${ }^{\circ}$ Brix) & $3,033 \pm 0,276$ \\
\hline Acidez titulable (\% ácido ascórbico) & $0,451 \pm 0,1648$ \\
\hline Contenido de humedad (\%) & $11,05 \pm 0,004$ \\
\hline Actividad de agua (Aw) & $0,410 \pm 0,015$ \\
\hline \multicolumn{2}{|c|}{ Coordenadas de color } \\
\hline $\mathrm{L}^{*}$ & $81,78 \pm 0,357$ \\
\hline$a^{*}$ & $18,5 \pm 0,128$ \\
\hline $\mathrm{b}^{*}$ & $23,2 \pm 0,640$ \\
\hline $\mathrm{C}$ & $29,7 \pm 0,185$ \\
\hline $\mathrm{h}$ & $51,4 \pm 0,056$ \\
\hline
\end{tabular}

$L^{*}: 0=$ negro y 100 = blanco; $a^{*}:-60=$ verde y $+60=$ rojo; $b^{*}:-60$ = azul y $+60=$ amarillo; Ángulo de tono (h): $90^{\circ}=$ amarillo, $180^{\circ}$ $=$ verde y $0^{\circ}=$ rojo; Cromaticidad $(\mathrm{C})$ : distancia desde las coordenadas en el origen hasta el punto de color determinado. Resultados reportados como media \pm desviación estándar para $n=3$. 
derante, siendo factor de aceptación o rechazo en la apariencia de dicho producto [39]. Los valores de las coordenadas L*, a* y b*, así como la cromaticidad $y$ el tono se muestran en el cuadro 1 . El valor alto de la luminosidad $\left(L^{*}\right)$, comparado con lo presentado para la pulpa de guayaba $\left(L^{*}=25,2\right)$ por Suárez et al. [40] y por Martínez-Ortiz et al. $\left(L^{*}=56,0\right)$ [33] da cuenta de una HEG relativamente oscura debido probablemente a la isomerización de los compuestos carotenoides y al proceso de pardeamiento causado por las temperaturas utilizadas durante el proceso de secado. Por otro lado, las coordenadas a* y b*, la cromaticidad (C) y el ángulo de tono (h) ligeramente superan lo presentado por Martínez-Ortiz et al. [33]. Sin embargo, estos valores son inferiores a lo mostrado por Formiga et al. [32], quienes trabajaron con guayaba roja "Pedro Sato". Las diferencias encontradas se deben, posiblemente, a las variedades de guayaba utilizadas en cada experimentación.

La actividad de agua junto con el contenido de humedad y el $\mathrm{pH}$ son parámetros determinantes en la validación de la vida útil de cualquier alimento. Los datos reportados en esta investigación permiten concluir que el contenido de humedad y la actividad de agua garantizan el no deterioro de la HEG ya que los valores son bajos y adecuados para un producto seco y molido, tal como lo afirman Lang et al. [41], quienes argumentan que dichos procesos de deterioro se ralentizan considerablemente a valores bajos de actividad de agua $(A w=0,60)$.

En el cuadro 2 se observan los resultados para el contenido de carotenoides en la HEG.

Se puede observar que el mayor valor se obtuvo para la fracción de $\beta$-criptoxantina, mientras que el menor valor fue para la fracción de $\alpha$-caroteno. Estos datos son superiores a los mostrados por Nora et al. [42], sin embargo, se registra igualmente, que para

Cuadro 2. Concentración de carotenoides en la harina de epicarpio de guayaba (HEG).

\begin{tabular}{|c|c|}
\hline Muestra & $\begin{array}{c}\text { Carotenoides en HEP } \\
\text { (mg/100 g) }\end{array}$ \\
\hline$\beta$-caroteno & $10,074 \pm 0,370$ \\
\hline a-caroteno & $9,414 \pm 0,859$ \\
\hline$\beta$-criptoxantina & $10,894 \pm 0,885$ \\
\hline Zeaxantina & $10,806 \pm 0,878$ \\
\hline Licopeno & $10,579 \pm 0,069$ \\
\hline
\end{tabular}

estos investigadores el mayor valor obtenido fue para la fracción de $\beta$-criptoxantina y el menor valor fue para el a-caroteno. Para Bordiga et al. [43] los valores obtenidos para los carotenoides trabajando con epicarpio y pulpa de la fruta caqui (Diospyros kaki L.) fueron supriores, al igual que los resultados mostrados por Sánchez-Camargo et al. quienes investigaron con cáscaras de mango [44] y para Noronha et al. quienes adelantaron su trabajo con cáscaras de tucumã (Astrocaryum vulgare) y chontaduro (Bactris gasipaes) [45]. Las diferencias presentadas entre las frutas analizadas y mostradas en la literatura, pueden deberse a diferentes factores, tales como los genéticos, los geográficos asociados a la producción de dichas frutas, las condiciones ambientales, grado de maduración, métodos de pre y poscosecha, etc.

El valor obtenido para la actividad antioxidante de la HEG fue de $62,281 \pm 1,519 \%$ (promedio \pm desviación estándar de tres repeticiones). Este valor fue menor que el mostrado por Kong e Ismail $(77,78 \%)$ [46] quienes trabajaron con subproductos de la producción de pulpa de guayaba y Murmu y Mishra $(84,45 \%)$ [47] quienes adelantaron su trabajo con un recubrimiento sobre la guayaba. Sin embargo, comparado con el valor referenciado por Nair, Saxena y Kaur $(40,2 \%)$ para guayaba recubierta con alginato y quitosano es mayor [48]. Respecto al contenido de compuestos fenólicos, el valor obtenido $(75,170 \pm$ $0,241 \mathrm{mg} E A G / g$ ), fue considerablemente menor a lo presentado por Murmu y Mishra (122,23 mg EAG/g) [47], así como también con relación a los resultados obtenidos por Nair, Saxena y Kaur (139,5 mg EAG/g) [48], los mostrados por Formiga et al. (271,2 mg EA$\mathrm{G} / \mathrm{g}$ ) quienes trabajaron con guayaba de la variedad "Pedro Sato" [32] y los aportados por Wolf y Cylos (682,29 mg EAG/g) quienes trabajaron con pasta de guayaba [49]. Por el contrario, los datos mostrados por Souza et al. (24,63 mg EAG/g) [50] quienes trabajaron con residuos industriales de guayaba, al igual que los encontrados por Ademiluyi et al. $(66,1 \mathrm{mg}$ EAG/g) [51] fueron menores.

Los datos obtenidos en el presente estudio, referenciados a los mostrados por otros autores en sus respectivas investigaciones, permiten afirmar que a mayor o menor concentración de compuestos fenólicos o de su grado de hidroxilación, se presenta una variación en la actividad de captación de radicales del DPPH y por lo tanto en la actividad antioxidante, debido a la dependencia que se presenta con relación a las pro- 
piedades de reducción y oxidación, las cuales pueden desempeñar un papel preponderante en la absorción y neutralización de los radicales libres. Por otro lado, el mayor o menor contenido de fenoles en la HEG tiene relación directa con las características genéticas de la variedad de guayaba utilizada y a su grado de maduración y condiciones agronómicas de producción [52].

\section{CONCLUSIONES}

Los valores obtenidos tanto para los parámetros fisicoquímicos como para las coordenadas del color y los contenidos de compuestos carotenoides y fenólicos y la actividad antioxidante de estos, permiten establecer que la harina de epicarpio de guayaba puede ser utilizada como fuente de pigmentos naturales para ser agregada a diversas matrices alimentarias. Además, gracias al alto contenido y a las propiedades funcionales de los componentes anotados presentes en la materia prima analizada, la HEG se muestra como una alternativa interesante, de origen natural, para enriquecer y prevenir enfermedades de alto riesgo para el ser humano.

\section{AGRADECIMIENTOS}

Los autores agradecen a la Universidad Nacional de Colombia - Sede Palmira por la financiación del proyecto HERMES 43547, de donde se deriva este artículo.

\section{REFERENCIAS}

[1] RAMOS, M., JIMÉNEZ, A. and GARRIGÓS, M.C. Il-Bassed advanced techniques of value-added compounds from natural sources and found by-products. Trends in Analytical Chemistry, 119, 2019, 115616. doi: https://doi. org/10.1016/j.trac.2019.07.027

[2] $\mathrm{BECH}, \mathrm{S}$. et al. Product and process variety management: case study in the food industry. Procedia CIRP, 81, 2019, p. 1065-1070. doi: https://doi.org/10.1016/j.procir.2019.03.252

[3] BUSSOLO DE SOUZA, C. et al. Characterization and in vitro digestibility of by-products from Brazilian food industry: Cassava bagasse, orange bagasse and passion fruit peel. Bioactive Carbohydrates and Dietary Fibre, 16, 2018, p. 90-99. doi: https:// doi.org/10.1016/j.bcdf.2018.08.001

[4] SPINELLI, S. et al. Food by-products to fortified pasta: A new approach for optimization. Journal of Cleaner Production, 215, 2019, p. 985-991. doi: https://doi.org/10.1016/j.clepro.2019.01.117

[5] LAFARGA P. T. V. et al. Revalorización de los subproductos de la industria de transformación de vegetales. Biblioteca Horticultura. Valencia (España): Serveis per la produccio editorial SPE3, 2018, $18 \mathrm{p}$.

[6] ROJAS-GARBANZO, C. et al. Characterization of phenolic and other polar compounds in peel and flesh of pink guava (Psidium guajava L. cv. "crioIla") by ultra-high performance liquid chromatography with diode array and mass spectrometric detection. Food Research International, 100(3), 2017, p. 445-453. doi: https://doi.org/10.1016/j. foodres.2016.12.004

[7] COLOMBIA. MINISTERIO DE AGRICULTURA Y DESARROLLO RURAL. Anuario estadístico del sector agropecuario 2016. Bogotá (Colombia): 2017, 290 p.

[8] NAGARAJAN, J. et al. A facile water-induced complexation of lycopene and pectin from pink guava byproduct: Extraction, characterization and kinetic studies. Food Chemistry, 296, 2019, p. 47-55. doi: https://doi.org/10.1016/j.foodchem.2019.05.135

[9] DA SILVA LIMA, R. et al. May de superfruit red guava and its processing waste be a potential ingredient in functional foods? Food Research International, 115, 2019, p. 451-459. doi: https:// doi.org/10.1016/j.fodres.2018.10.053

[10] CHANG, S.K., ALASALVAR, C. and SHAHIDI F. Superfruits: Phytochemicals, antioxidant, efficacies, and health effects - A comprehensive review. Critical Reviews in Food Science and Nutrition, 59(10), 2019, p. 1580-1604. doi: https://doi.org /10.1080/10408398.2017.1422111

[11] KATIA, C.R. et al. Residuos agroindustriales su impacto, manejo y aprovechamiento. Revista Colombiana de Ciencia Animal, 9, 2017, p. 122-132. doi: https://doi.org/10.24188/recia.v9.nS.2017.530

[12] SERNA-COCK, L., MERA-AYALA, J.D. and ANGULO-LÓPEZ, J.E. Guava Psidium guajava seed flour and dry Aspergillus niger mycelium as nitrogen sources for the Production of biomass and antimicrobial compounds produced by Weissella confusa. Electronic Journal of Biotechnology, 16(6), 2013, p. 1-9. doi: https://doi.org/10.2225/vol16-issue6-fulltext-1 
[13] MARTÍNEZ-GIRÓN, J.y ORDÓÑEZ-SANTOS, L.E. Determinación de la concentración de pigmentos carotenoides en harina de residuos de chontaduro (Bactris gasipaes). Revista Producción + Limpia, 11(1), 2016, p. 85-93.

[14] BATISTA, P.F. et al. Bioactive compounds and antioxidant activity in guava fruit cultivated in Sub-Middle São Francisco Valley, Brazil. Abstracts $3^{\text {rd }}$ International Symposium on Guava and Other Myrtaceae, Petronila, PE (Brasil): Embrapa, 2012, p. 51-52.

[15] ORDÓÑEZ-SANTOS, L.E. et al. Concentración de carotenoides totales en residuos de frutas tropicales. Producción + Limpia, 9(1), 2014, p. 91-98.

[16] MERHAN, O. En: Carotenoids. The biochemistry and antioxidant properties of carotenoids. 3 ed. London (England): Intechopen, 2017, p. 50-66

[17] MARTíNEZ, G.J. et al. Pigmentos vegetales y compuestos naturales aplicados en productos cárnicos como colorantes y/o antioxidantes: revisión. Inventium, 11(21), 2016, p. 51-62. doi: https://doi.org/10.26620/uniminuto.inventarium.11.21.2016.51-62

[18] NGBOLUA, K.N. et al. A review on the phytochemistry and pharmacology of Psidium guajava L. (Myrtaceae) and future direction. Discovery Phytomedicine, 5(2), 2018, p. 7-13. doi: https:// doi.org/10.15562/phytomedcine.2018.58

[19] LOPEZ DOS SANTOS, W.N. et al. Simultaneous determination of 13 phenolic bioactive compounds in guava (Psidium guajava L.) by HPLCPAD with evaluation using PCA and Neural Network Analysis (NNA). Microchemical Journal, 133, 2017, p. 583-592. doi: https://doi.org/10.1016/j.microc.2017.04.029

[20] ROJAS-GARBANZO, C. et al. Characterization of phytochemicals in Costa Rican guava (Psidium friedrichsthalianum-Nied.) fruit and stability of main compounds during juice processing - (U)HPLC-DAD-ESI-TQD-MSn. Journal of Food Composition and Analysis, 75, 2019, 26-42. doi: https://doi.org/10.1016/jfca.2018.09.012

[21] BLANCAS-BENITEZ, F.J. et al. In vitro evaluation of the kinetics of the release of phenolic compounds from guava (Psidium guajava L.) fruit. Journal of Functional Foods, 43, 2018, p. 139-145. doi: https://doi.org/10.1016/j.jff.2018.02.011

[22] MAJOU, D. and CHRISTIEANS, S. Mechanisms of the bactericidal effects of nitrate and nitrite in cured meats. Meat Science, 145, 2018, p. 273-284. doi : https://doi.org/10.1016/j.meatsci.2018.06.013

[23] CARDINALLI, F. et al. Microbial dynamics of model Fabriano-like fermented sausages as affected by starter cultures, nitrates and nitrites. International Journal of Food Microbiology, 278, 2018, p. 61-72. doi: https://doi.org/10.1016/j. ijfoodmicro.2018.04.002

[24] HAMDI, M. et al. Improvement of the quality and the shelf life of reduced-nitrites turkey meat sausages incorporated with carotenoproteins from crabs shells. Food Control, 91, 2018, p. 148-159. doi: https://doi.org/10.1016/j.foodcont.2018.03.048

[25] FLORES, M. et al. Risk assessment of chemical substances of safety concern generated in processed meats. Food Science and Human Wellness, Avalaible online 29 July 2019 ,. doi: https://doi. org/10.1016/j.fshw.2019.07.003

[26] INSTITUTO COLOMBIANO DE NORMAS TÉCNICAS (ICONTEC). NTC 4592, NTC 4624, NTC 4623: Fruit and vegetable products. Determination of $\mathrm{pH}$, Determination of soluble solids content, Determination of Titrable Acidity. Bogotá (Colombia): 1999, 21 p.

[27] ASSOCIATION OF CHEMICAL ANALYTICAL CHEMISTS (AOAC). Official Methods of Analysis, Methods 925.09: Moisture in cassava - air oven methods. Washington D.C. (USA): 2005.

[28] ASSOCIATION OF CHEMICAL ANALYTICAL CHEMISTS (AOAC). Official Methods of Analysis, Methods 978.19. Maryland (USA): 2000.

[29] MARTÍNEZ-GIRÖN, J. et al. Caracterización fisicoquímica de harina de residuos del fruto de chontaduro (Bactris gasipaes Kunth Arecaceae) obtenida por secado convectivo. Ciencia y Tecnologia Agropecuaria, 18(3), 2017, p. 599-613. doi: https:// doi.org/10.21930/rcta.vol18_num3_art:747

[30] SINGLETON, V.L., ORTHOFER, R. and RAVENTÓS, R.M. Analysis of total phenols and other oxidation substrates and antioxidants by means of Folin-Ciocalteu reagent. Methods in Enzymology, 299, 1999, p. 152-178. doi: https://doi. org/10.1016/S0076-6879(99)99017-1

[31] VASCONCELOS, M.C.E. et al. Fruits from Brazilian Cerrado region: Physico-chemical characterization, bioactive compounds, antioxidant activities, and sensory evaluation. Food Chemistry, 245, 2018, p. 305-311. doi: https://10.1016/j. foodchem.2017.10.104 
[32] FORMIGA, A.S. et al. Use of edible coatings based on hydroxypropyl methylcellulose and beeswax in the conservation of red guava "Pedro Sato". Food Chemistry, 290, 2019, p. 144-151.

[33] MARTÍNEZ-ORTIZ, M.A. et al. Effect of using microencapsulated ascorbic acid in coatings based on resistant starch chayotextle on the quality of guava fruit. Scientia-Horticulturae, 256, 2019, 108604. doi: https://doi.org/j.scienta.2019.108604

[34] ZAMBRANO-ZARAGOZA, M.L. et al. Use of solid Lipid nanoparticles (SLNs) in edible coatings to increase guava (Psidium guajava L.) shelf-life. Food Research International, 51(2), 2013, p. 946-953. doi: https://doi.org/10.1016/j.foodres.2013.02.012

[35] ALAMAR, P.D. et al. Quality evaluation of frozen guava and yellow passion fruit pulps by NIR spectroscopy and chemometrics. Food Research International, 85, 2016, p. 209-214. doi: https://doi.org/10.1016/j.foodres.2016.04.027

[36] MOON, P. et al. Assessment of fruit aroma for twenty-seven guava (Psidium guajava) accessions through three fruit developmental stages. Scientia Horticulturae, 238, 2018, p. 375-383. doi: https:// doi.org/10.1016/j.scienta.2018.04.067

[37] MURMU, S.B. and MISHRA, H.N. Selection of the best active modified atmosphere packaging with ethylene and moisture scavengers to maintain quality of guava during low-storage. Food Chemistry, 253, 2018, p. 55-62. doi: https://10.1016/j.foodchem.2018.01.134

[38] SILVA, W.B. et al. Chitosan delays ripening and ROS Production in guava (Psidium guajava L.) fruit. Food Chemistry, 242, 2018, p. 232-238. doi: https://10.1016/j.foodchem.2017.09.052

[39] PAAKKI, M. et al. The importance of the visual aesthetics of colours in food at a workday lunch. International Journal of Gastronomy and Food Science, 16, 2019, 100131. doi: https:// doi.org/10.1016/j.ijgfs.2018.12.001

[40] SUAREZ, C.S. et al. Ultrasound processing of guava juice: effect on structure, physical properties and lycopene in vitro accessibility. Food Chemistry, 268, 2018, p. 594-601. doi: https:// doi.org/10.1016/j.fodchem.2018.06.127

[41] LANG, E. et al. Modeling the heat inactivation of foodborne pathogens in milk powder: high relevance of the substrate water activity. Food Research International, 99(1), 2017, p. 577-585. doi: https:// doi.org/10.1016/j.foodres.2017.06.028
[42] NORA, C.D. et al. Effect of processing on the stability of bioactive compounds from red guava (Psidium cattleyanum Sabine) and guabiju (Myrcianthes pungens). Journal of Food Composition and Analysis, 34(1), 2014, p. 18-25. doi: https://doi.org/10.1016/j.jfca.2014.01.006

[43] BORDIGA, M. et al. Characterization of peel and pulp proanthocyanidins and carotenoids during ripening in persimmon "Kaki Tipo" cv, cultivated in Italy. Food Research International, 120, 2019, p. 800-809. doi: https://doi.org/10.1016/j.foodres.2018.11.041

[44] SÁNCHEZ-CAMARGO, A.P. et al. Valorization of mango peel: proximate composition, supercritical fluid extraction of carotenoids, and application as an antioxidant additive for an edible oil. The Journal of Supercritical Fluids, 152, 2019, 104574. doi: https://doi.org/10.1016/j.supblu.2019.104574

[45] NORONHA, K. A. et al. Peels of tucumã (Astrocaryum vulgare) and peach palm (Bactris gasipaes) are by-products classified as very high carotenoid sources. Food Chemistry, 272, 2019, p. 216-221. doi: https://doi.org/10.1016/j.foodchem.2018.08.053

[46] KONG, K.W. and ISMAIL, A. Lycopene content and lipophilic antioxidant capacity of by-products from Psidium guajava fruits produced during puree production industry. Food and Bioproducts Processing, 89(1), 2011, p. 53-61. doi: https://doi.org/10.1016/j.fbp.2010.02.004

[47] MURMU, S.B. and MISHRA, H.N. The effect of edible coating based on Arabic gum, sodium caseinate and essential oil of cinnamon and lemon grass on guava. Food Chemistry, 245, 2018, p. 820-828.

[48] NAIR, M.S., SAXENA, A. and KAUR, C. Effect of chitosan and alginate based coatings enriched with pomegranate peel extract to extend the postharvest quality of guava (Psidium guajava L.). Food Chemistry, 240, 2018, p. 245-252. doi: https:// doi.org/10.1016/j.foodchem.2017.07.122

[49] WOLF, A.P. and CYLOS, C.M. Effect of industrial processing for obtaining guava paste on the antioxidant compounds of guava (Psidium guajava L.) "Paluma" cv. Revista Brasileira de Fruticultura, 40(2), 2018, 1-11. doi: https://doi. org/10.1590/0100-29452018011

[50] SOUZA, M.S.B. et al. Caracterização nutricional e compostos antioxidantes em resíduos de polpas de frutas tropicais. Ciencia e Agrotecnologia, 35(3), 2011, p. 554-559. doi: https://doi. org/10.1590/S1413-70542011000300017 
[51] ADEMILUYI, A.O. et al. A comparative study on antihypertensive and antioxidant properties of phenolic extracts from fruit and leaf of some guava (Psidium guajava L.) varieties. Comparative Clinical Pathology, 25(2), 2016, p. 363-374. doi: https:// doi.org/10.1007/s00580-015-2192-y

[52] VASCO, C., RUALES, J. and KAMAL-ELDIN, A. Total phenolic compounds and antioxidant capacities of major fruits from Ecuador. Food Chemistry, 111(4), 2008, p. 816-823. doi: https://doi.org/10.1016/j.foodchem.2008.04.05 